\title{
Regional impairment of cerebral oxidative metabolism in Parkinson's disease
}

\author{
G . L. LE N Z I, T. JO NES, J L . R E I D, A N D S . MOS S \\ From the MRC Cyclotron Unit, Departments of Medical Physics and Clinical Pharmacology, \\ Royal Postgraduate Medical School, Hammersmith Hospital, London, and Institute of Neurology, \\ University of Rome, Italy
}

S U MMARY Twenty-two patients affected by idiopathic Parkinsonism were studied using the oxygen-15 inhalation technique. The production of labelled metabolic water was found to be decreased in the parietal cerebral cortex, indicating an impairment in oxidative metabolism. This metabolic defect was localised mainly to the parietal cortex of the affected hemispheres; in non-affected hemispheres of patients presenting with unilateral Parkinsonism, the uptake was normal. In contrast, regional blood flow was not significantly altered. It is possible that this metabolic impairment is caused by chronic deafferentation.

It has been established that Parkinson's disease is caused by biochemical-metabolic changes which result in an impairment of the normal neurotransmitter balance in the basal ganglia, substantia nigra, and other cerebral regions. It seemed appropriate, therefore, to examine, in life, the full extent of the functional defects in regional cerebral metabolic activity. Such an investigation could provide insight into the overall effects on regional cerebral function and may have implications for the early diagnosis of the disease, and for the development and assessment of therapeutic agents. To this end, a recently developed non-invasive technique using inhalation of radioactive oxygen15 for the assessment of regional oxygen utilisation and blood flow in the human brain (Jones et al., 1976; Lenzi et al., 1978a) was applied to a group of patients with Parkinsonism. The working hypothesis was that the alteration of neurotransmitter metabolism could be reflected by a modification of local oxygen utilisation and blood flow.

The study of unilateral Parkinson's disease offered the opportunity to obtain control records from the same patients, by comparing the affected hemisphere with the contralateral (non-affected) one. The non-invasive nature of the inhalation technique allowed a longitudinal follow-up, and in addition enabled a comparison to be made with the results obtained from a normal control group.

Address for reprint requests: Dr T. Jones, MRC Cyclotron Unit, Hammersmith Hospital, Du Cane Road, London W12 OHS.

Accepted 14 July 1978

\section{Methods}

The basic principles of the technique and the results obtained in normal subjects and neurological patients have been presented previously (Lenzi et al., 1978a,b). The oxygen-15 inhalation technique takes advantage of the short half-life of oxygen-15 (2.1 minutes) and the relatively high uptake of oxygen by the nervous tissue. A Gamma camera recording of the regional radioactivity within the brain when a steady state period has been reached indicates the local production of labelled metabolic water-that is, aerobic metabolism. The same recording while the patient inhales labelled $\mathrm{C}^{15} \mathrm{O}_{2}$ at physiological concentrations, reflects the transport of labelled water and, in turn, the regional perfusion of the brain. Hence, the inhalation procedures provide two cerebral distributions which relate to the regional oxygen consumption and the regional blood flow. The regional values within the metabolic distribution are expressed as being the metabolic ratios (MR) and those of the circulatory distribution as perfusion ratios (PR). The point-to-point ratio of the two distributions (MR/ $\mathrm{PR}$ ) indicates the regional oxygen extraction ratio (OER) of the brain tissue.

\section{Subjects}

Twenty-two patients with idiopathic Parkinsonism were studied, 14 men and eight women. Their mean age was $68 \pm 9$ years, and the duration of the 
disease ranged from one to 17 years (mean $5 \pm 4$ ). No signs of dementia were reported.

In nine patients the disease was completely unilateral, in seven predominantly unilateral, and in six bilateral.

Fourteen cases were treated with levodopa combined with peripheral decarboxylase inhibitors, one case received levodopa alone, and in four benzhexol was given together with a levodopa preparation. The severity of the disease was evaluated by a fixed point rating, with four levels for the three main symptoms: tremor, rigidity, and akinesia. In the rating, the score 0 represents absence of symptoms, and 4 the maximal disability (Vakil et al., 1973). A total of 25 affected hemispheres were studied.

As controls, besides using non-affected hemispheres, 10 normal volunteers, with a mean age of $56 \pm 7$ years, were studied. The necessity of comparing patient groups of similar age is particularly relevant in studies on metabolic activity, since modifications with aging have been observed (Lenzi and Jones, unpublished).

\section{Results}

During the metabolism and circulation procedures, the distribution of activity recorded in the central regions of the brain-that is, in those parts corresponding to the basal ganglia-was not significantly different in Parkinsonism patients as com- pared with normal volunteers. However, focal reductions were seen in the cortical regions, with the parietal area predominantly involved. On analysing the relative quantitative uptake of radioactivity in the parietal region, we found that the metabolic ratio (MR) was affected more than the perfusion ratio (PR). This in turn resulted in a significant $(P<0.05)$ reduction of the oxygen extraction ratio (OER) as shown in the Table.

Motor regions also showed a decreased metabolic activity in affected and in non-affected hemispheres, as compared with controls. Circulatory activities were within normal limits. Figure

Table Mean values $\pm S D$ obtained for the oxygen extraction (OER), metabolic (MR), and perfusion ratios $(P R)$ in the different cortical regions in the three groups studied

\begin{tabular}{|c|c|c|c|c|}
\hline \multicolumn{2}{|c|}{ Regional values } & \multirow{2}{*}{$\begin{array}{l}\begin{array}{l}\text { Parkinsonism } \\
(\text { affected }) \\
(24 \text { cases })\end{array} \\
\begin{array}{c}0.89 \pm 0.05 \\
69.10 \pm 4.80 \\
77.00 \pm 4.40\end{array}\end{array}$} & \multirow{2}{*}{$\begin{array}{c}\begin{array}{c}\text { Parkinsonism } \\
(\text { not affected }) \\
(12 \text { cases })\end{array} \\
0.98 \pm 0.05 \\
73.50 \pm 4.30 \\
75.20 \pm 4.10\end{array}$} & \multirow{2}{*}{$\begin{array}{l}\begin{array}{l}\text { Control } \\
\text { subjects } \\
(10 \text { cases })\end{array} \\
0.98 \pm 0.06 \\
76.40 \pm 2.60 \\
79.00 \pm 3.10\end{array}$} \\
\hline Parietal & $\begin{array}{l}\text { OER } \\
\text { MR } \% \\
\text { PR } \%\end{array}$ & & & \\
\hline Motor & $\begin{array}{l}\text { OER } \\
\text { MR } \% \\
\text { PR } \%\end{array}$ & $\begin{array}{r}0.95 \pm 0.07 \\
73.00 \pm 6.10 \\
76.90 \pm 4.40\end{array}$ & $\begin{array}{r}0.95 \pm 0.06 \\
74.80 \pm 6.40 \\
77.60 \pm 4.60\end{array}$ & $\begin{array}{r}1.01 \pm 0.05 \\
78.40 \pm 3.20 \\
79.00 \pm 4.30\end{array}$ \\
\hline Occipital & $\begin{array}{l}\text { OER } \\
\text { MR } \% \\
\text { PR } \%\end{array}$ & $\begin{array}{r}1.00 \pm 0.06 \\
76.30 \pm 6.10 \\
76.60 \pm 4.30\end{array}$ & $\begin{array}{r}1.01 \pm 0.05 \\
79.70 \pm 4.20 \\
78.50 \pm 4.70\end{array}$ & $\begin{array}{r}0.99 \pm 0.06 \\
79.50 \pm 8.30 \\
78.60 \pm 2.30\end{array}$ \\
\hline Frontal & $\begin{array}{l}\text { OER } \\
\text { MR } \% \\
\text { PR } \%\end{array}$ & $\begin{array}{r}0.99 \pm 0.07 \\
74.90 \pm 5.70 \\
74.60 \pm 6.40\end{array}$ & $\begin{array}{r}0.98 \pm 0.06 \\
73.90 \pm 3.00 \\
75.60 \pm 2.80\end{array}$ & $\begin{array}{r}1.03 \pm 0.06 \\
76.00 \pm 7.30 \\
75.50 \pm 5.90\end{array}$ \\
\hline
\end{tabular}

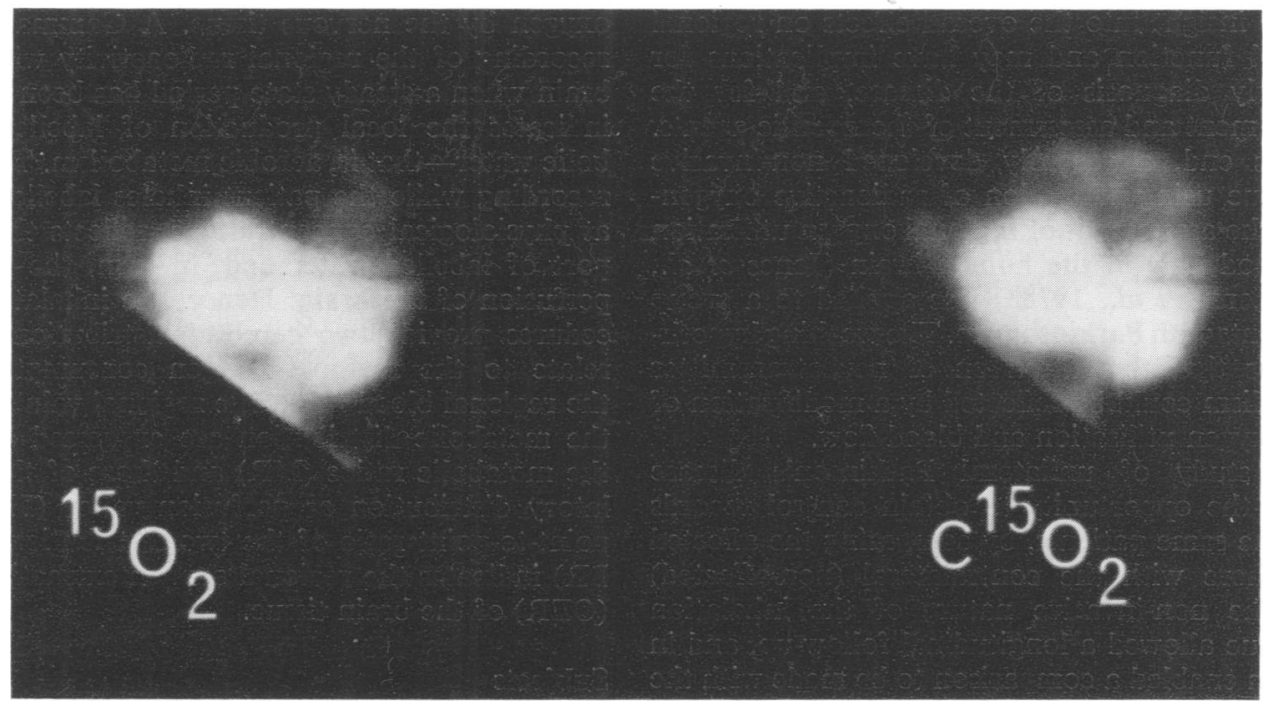

Fig. 1 Left lateral views of cerebral distributions of oxygen uptake $\left({ }^{15} \mathrm{O}_{2}\right.$, left $)$ and circulation $\left(C^{15} O_{2}\right)$ in a patient affected by right sided Parkinsonism. A large decrease of metabolic activity is seen in the left scan, while in the right scan only the frontal region shows a parallel defect of perfusion. 
1 shows an example of a patient presenting with a diffuse reduction of metabolic activity in frontal, motor, and parietal regions. In this patient, circulatory activity for the motor and parietal regions were not significantly different from controls.

The depression of the parietal oxygen extraction ratio is caused by an imbalance between metabolism and blood flow. The circulatory activity was within normal limits in almost $50 \%$ of the affected hemispheres while only in $18 \%$ was the metabolic activity in the affected parietal region within normal limits. In the non-affected hemispheres, the metabolic and circulatory activities were in close correlation, demonstrating the normal link between metabolism and blood flow for the same region of the brain (Fig. 2).

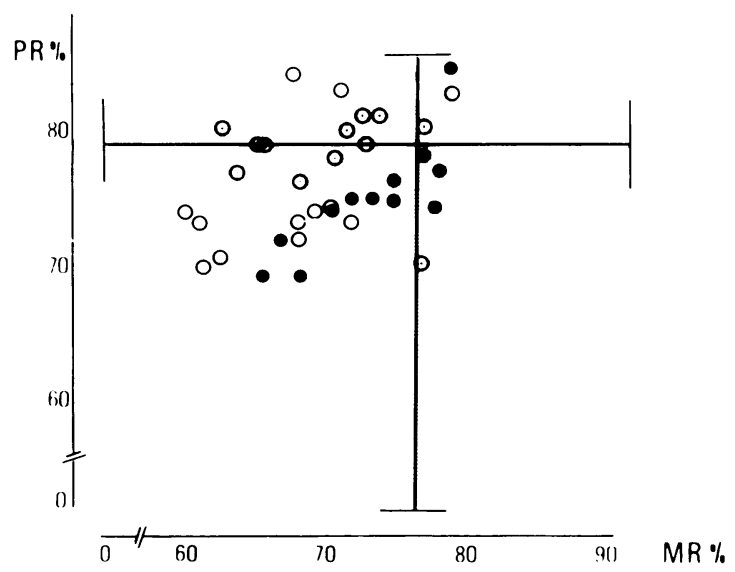

PARIETAL REGION

Fig. 2 Correlation between metabolic and perfusion ratios in 24 affected $(O)$ and 12 non-affected $(O)$ parietal regions. Vertical line indicates the mean metabolic ratio for the control group, with the standard deviation. Horizontal line indicates the mean perfusion ratio for the control group, with the standard deviation. Note the close correlation, for the non-affected parietal regions, between the metabolic ratio and the perfusion ratio.

No correlation was found between the decrease in the parietal oxygen extraction ratio and the severity of the disease, evaluated with a fixed point rating (Fig. 3). On the other hand, a statistically significant relationship was found between the duration of the disease and the reduction of the OER in the parietal area $(P<0.05)$ (Fig. 4).

In eight patients the examination was repeated after a period ranging between five and 17 months; the mean values were in agreement with the previous observations.

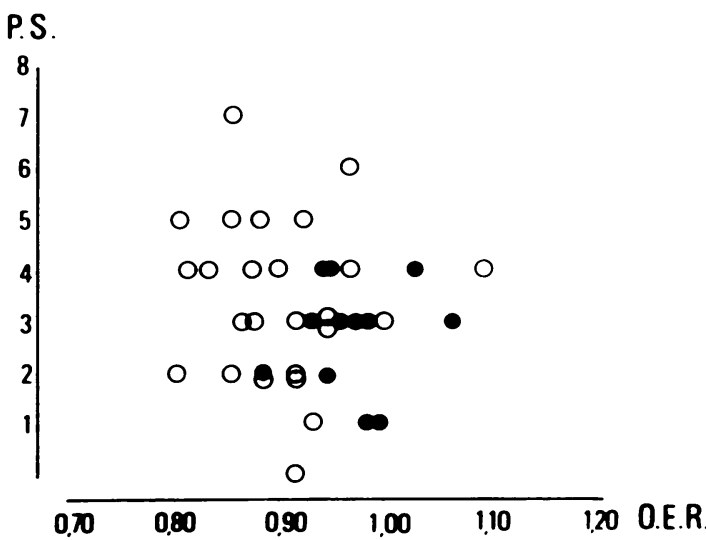

\section{PARIETAL REGION}

Fig. 3 Correlation between the oxygen extraction ratio in the parietal region and the severity of the disease (symbols as in Fig. 2). Statistical analysis did not show any significant correlation between the variables.

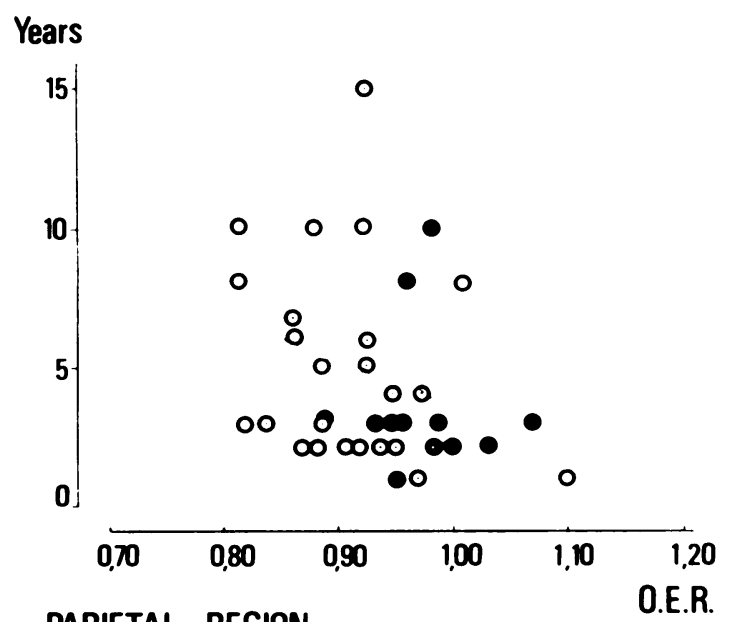

PARIETAL REGION

Fig. 4 Correlation between the parietal $O E R$ and duration of the disease (years), in the affected and non-affected hemisphere (symbols as in Fig. 2). Statistical analysis showed a slightly significant correlation $(P<0.05)$ between the two parameters in the affected hemispheres. No correlation was found for the non-affected hemispheres.

\section{Discussion}

The study of a group of patients affected by Parkinson's disease with the non-invasive oxygen15 inhalation technique has demonstrated an impairment in the oxidative metabolism predominantly within the parietal cortex of the brain. 
Other cerebral regions (frontal and occipital), or the corresponding contralateral regions in cases of unilateral Parkinson's disease, were statistically unaffected. In the motor regions there was a bilateral reduction in metabolic activity, but the OER was not significantly depressed.

This regional impairment in metabolic activity may either be secondary to chronic deafferentation, because of a minor physiological activity in the thalamo-parietal pathways, or could be a primary deficit. The former hypothesis seems the most probable. However, although the more striking histological and biochemical deficit in Parkinsonism is the degeneration of the nigrostriatal dopaminergic pathways and the loss of dopamine from the striatum, it has long been recognised that other pathways are functionally if not anatomically impaired in Parkinsonism (Pletscher, 1976). Several other neurotransmitters (serotonin, GABA, substance $P$, and so on) in addition to dopamine, have been proposed as participating in extrapyramidal motor control. The primary biochemical defect may initiate a chain of other biochemical and functional impairments.

There is, therefore, the possibility that the metabolic impairment within the parietal cortex may represent an example of diaschisis caused by transneuronal depression (Ginsberg et al., 1977). Previous authors have stressed that the sensorimotor cortex may also be involved early in the disease (Selby, 1968), and sensorimotor control is depressed in patients presenting with Parkinsonism. This provides evidence of a central processing deficit (Horne, 1973) that may be the basis of the negative symptoms (akinesia) of the Parkinson's disease. Angel et al. (1970) suggest a disorder at, or very near, the highest level of the motor system in the akinetic patients. In the present study, patients with higher scores for akinesia showed, as a group, the lower metabolic activities within their parietal cortex. It is unlikely that drug therapy was responsible for the observed changes as metabolic activity was reduced in the cortical areas of the affected hemisphere in unilateral Parkinson's disease. In addition, no differences were found between patients treated with different drugs.

These observations of a functional deficit in the central nervous system at a site distant to the localised area of the neurological lesion illustrate the usefulness of this non-invasive technique. The method is currently limited to two-dimensional imaging. Hence, it was not possible to analyse metabolic activity within the central regions of the nervous system, because of the superimposition of other cerebral structures. This degree of analysis could be possible if three-dimensional tomographic devices were to be used (Reivich et al., 1977).

We thank Professor C. Fieschi for his suggestions and criticism in the preparation of the manuscript. The technical support of Mr P. J. Sleight, Mr P. D. Buckingham, and the Cyclotron operating team is recognised as being of utmost importance. The research has been partly supported by the International Grant of the CNR, Italy, No. 770146804.

\section{References}

Angel, R. A., Alsten, W., and Higgins, J. R. (1970). Control of movement in Parkinson's disease. Brain, 93, 1-15.

Ginsberg, M. D., Reivich, M., Giandomenico, A., and Greenberg, J. H. (1977). Local glucose utilization in acute focal cerebral ischemia: local dysmetabolism and diaschisis. Neurology (Minneapolis), 27, 10421048.

Horne, D. J. de L. (1973). Sensorimotor control in Parkinsonism. Journal of Neurology, Neurosurgery, and Psychiatry, 36, 742-746.

Jones, T., Chesler, D. A., and Ter-Pogossian, M. M (1976). The continuous inhalation of oxygen-15 fo assessing regional oxygen extraction in the brain of man. British Journal of Radiology, 49, 339-343.

Lenzi, G. L., Jones, T., McKenzie, C. G., Buckinghamg P. D., Clark, J. C., and Moss, S. (1978a). A study of regional cerebral metabolism and blood flow reO lationships in man using the method of continus ously inhaling oxygen-15 and oxygen-15 labellew carbon dioxide. Journal of Neurology, Neurosurgery and Psychiatry, 41, 1-10.

Lenzi, G. L., Jones, T., McKenzie, C. G., and Moss, S. (1978b). A non-invasive regional study of chronic cerebrovascular disorders using the oxygen-15 inhalation technique. Journal of Neurology, Neurosurgery, and Psychiatry, 41, 11-17.

Pletscher, A. (1976). Biochemical and pharmacological aspects of Parkinson's syndrome. In Advances in Parkinsonism, pp. 21-36. Edited by W. Birkmayer and O. Hornykiewicz. Editions Roche: Basel.

Reivich, M., Kuhl, D., Wolf, A., Greenberg, J., Phelps, M., Ido, T., Casella, V., Fowler, J., Gallagher, B., Hoffman, E., Alavi, A., and Sokoloff, L. (1977). Measurement of local cerebral glucose metabolism in man with 18F-2-fluoro-2-deoxy-D-glucose. Acta Neurologica Scandinavica, Supplement 64, 190-191.

Selby, G. (1968). Parkinson's disease. In Handbook of Clinical Neurology, vol. 6, pp. 181-211. Edited by P. J. Vinken and G. W. Bruyn. North-Holland: Amsterdam.

Vakil, S. D., Calne, D. B., Reid, J. L., and Seymour, C. A. (1973). Pyrimidyl-piperazine (ET 495) in Parkinsonism. In Progress in Treatment of Parkinsonism, pp. 121-125. Raven Press: New York. 